\title{
Quercetin alleviates generalized hyperalgesia in mice with induced adenomyosis
}

\author{
JICHAN NIE and XISHI LIU \\ Department of Gynecology, Obstetrics and Gynecology Hospital of Fudan University, \\ Fudan University, Shanghai 200011, P.R. China
}

Received October 22, 2016; Accepted June 8, 2017

DOI: $10.3892 / \mathrm{mmr} .2017 .7238$

\begin{abstract}
Adenomyosis is a common gynecologic disorder characterized by the presence of endometrial glands and stroma within the myometrium. The present study investigated the effect of quercetin in neonatal Imprinting Control Region mice with tamoxifen-induced adenomyosis. The body weight and hotplate response latency of all mice was examined at 4, 8, 12 and 16 weeks after birth. The mice dosed with tamoxifen were divided into four groups: high- or low-quercetin group, valproic acid (VPA) group and untreated group. The group of mice that were neonatally administrated with the solvent only (no tamoxifen), received no treatment and served as a blank control group. After 3 weeks of drug treatment, the potential ability of quercetin to improve the generalized hyperalgesia in mice with induced adenomyosis was evaluated by determining the body weight, pain modulation, examining the myometrial infiltration by histology examination of the uterus and detecting the expression of transient receptor potential cation channel subfamily $\mathrm{V}$ member 1 (Trpv-1), phospho (p)-p38 mitogen activated protein kinase p-extracellular signal-regulated kinase (p-ERK) in DRG neurons via immunohistochemistry. The results demonstrated that treatment with quercetin improved the generalized hyperalgesia by extending the hotplate response latency, reduced myometrial infiltration and decreased the expression levels Trpv-1, p-p38 and p-ERK in dorsal root ganglion neurons. The results indicated that quercetin decreases the incidence of hyperalgesia in mice with tamoxifen-induced adenomyosis, and the potential mechanism is through reduced central sensitization, which may be a promising treatment for adenomyosis.
\end{abstract}

\section{Introduction}

Adenomyosis is a common gynecological disorder characterized by heterotopic endometrial glands and stroma in

Correspondence to: Dr Xishi Liu, Department of Gynecology, Obstetrics and Gynecology Hospital of Fudan University, Fudan University, 419 Fangxie Road, Shanghai 200011, P.R. China

E-mail: liuxishish@163.com

Key words: adenomyosis, hyperalgesia, mouse, quercetin myometrium. It frequently affects women aged 30-50 years (1) and often causes dysmenorrhea, menorrhagia, metrorrhagia, diffusely enlarged uterus, and dyspareunia, as well as infertility $(2,3)$. Yet the exact mechanisms of adenomyosis-induced pain are still unknown. Therefore, treating adenomyosis is a major challenge. Currently, surgical and medical therapies are the common treatment of adenomyosis. For severe, symptomatic adenomyosis, hysterectomy is the treatment choice (4), which can be traumatic for women wishing to conceive. Consequently, medical therapies, such as danazol and gonadotropin-releasing hormone agonist, are important for patients aiming to become pregnant. However, evidence has showed that there are several limitations of these treatments, including the long period of treatment, severe adverse events and frequent recurrence (5). Therefore, further studies on developing more efficacious medical therapies are required.

Quercetin (3,3',4',5,7-pentahydroxyflavone; molecular weight, $302 \mathrm{~g} / \mathrm{mol}$ ), a bioactive flavonoid is widely present in various fruits and vegetables, including onions, and the most studied quercetin primary sources are broccoli, apples, berry crops and grapes, and is also in certain herbs, tea and wine $(6,7)$. Emerging evidence demonstrated that quercetin has potential benefits for treatment of cancer and endometriosis with no obvious adverse actions, with anti-carcinogenic, anti-inflammatory, anti-viral, anti-oxidant, and psychostimulant activities, as well as the ability to inhibit lipid peroxidation, platelet aggregation and capillary permeability, and to stimulate mitochondrial biogenesis $(8,9)$. However, the underlying mechanism of quercetin alleviating generalized hyperalgesia of adenomyosis remains poorly understood. Therefore, well-designed trials to evaluate the effect of quercetin on adenomyosis are required.

The objective of this study as to evaluate the effect of quercetin in Imprinting Control Region (ICR) mice with adenomyosis. It was aimed to investigate whether the treatment of quercetin improve pain and suppress the myometrial infiltration of endometrial implants. We hypothesized that quercetin may reduce hyperalgesia and also suppress myometrial infiltration of ectopic endometrium in mice with induced adenomyosis. In order to elucidate the molecular mechanism of quercetin treatment, the levels of certain important mediators in pain, including transient receptor potential cation channel subfamily V member 1 (Trpv-1), phospho (p)-p38 mitogen-activated protein kinase (MAPK) and p-extracellular 
signal-regulated kinase (ERK), were determined. Our previous study demonstrated that valproic acid (VPA), a histone deacetylase inhibitor, improved generalized hyperalgesia and reduced the myometrial infiltration (10). Therefore, VPA was used as a positive indicator in the present study.

\section{Materials and methods}

Chemicals. Quercetin and VPA (VPA sodium salt, cat. no. P4543) were purchased from Sigma-Aldrich (Merck KGaA, Darmstadt, Germany). All of these drugs were dissolved in $2 \%$ dimethyl sulphoxide in $0.1 \mathrm{M} \mathrm{Na}_{2} \mathrm{SO}_{4}$ adjusted to $\mathrm{pH}$ 5.0. Tamoxifen citrate was purchased from Fudan Forward Pharmaceutical Company (Shanghai, China).

Animals and treatments. Newborn ICR mice (day 1 after birth; $\mathrm{n}=43)$ and their birth mother mice $(\mathrm{n}=10)$ were purchased from Shanghai Laboratory Animal Corporation (Shanghai, China). Each mother mouse and her offspring were housed in one cage under controlled conditions $\left(\sim 20^{\circ} \mathrm{C}, 12: 12\right.$ light/dark cycle) and had free access to food and fresh water.

Following the protocol of Green et al (11) and Parrott et al (12), female neonatal mice were orally administered $1 \mathrm{mg} / \mathrm{kg}$ tamoxifen suspended in peanut oil/lecithin/condensed milk mixture (2:0.2:3, by volume) at a dose volume of $5 \mu \mathrm{l} / \mathrm{g}$ body weight from day 2 to day 5 after birth. Female control neonatal mice $(n=9)$, designated randomly, were fed with the equivalent amount of solvent, without tamoxifen. When mice were 3 weeks old, they were weaned and separated from their mothers, and female mice continued as part of the study.

All experiments were performed under the guidelines of the National Research Council's Guide for the Care and Use of Laboratory Animals (13) and approved by the institutional experimental animals review board of Shanghai Obstetrics and Gynecology Hospital, Fudan University (Shanghai, China).

Experiment protocol. Neonatal mice dosed with tamoxifen as described $(n=34)$ and 9 control mice were dosed with solvent only. Starting from 4 weeks after birth, a hotplate test was administrated to all mice every 4 weeks, and their body weight was also measured and recorded. At 16 weeks after birth, all mice dosed with tamoxifen were randomly divided into 4 groups, each receiving a certain treatment by daily intragastric administration for 3 weeks as follows: Group L $(\mathrm{n}=9)$ received a low-dose $(25 \mathrm{mg} / \mathrm{kg}$ body weight $)$ quercetin treatment; Group $\mathrm{H}(\mathrm{n}=9)$ received a high-dose $(50 \mathrm{mg} / \mathrm{kg}$ body weight) quercetin treatment; Group V $(n=8)$ received VPA treatment (160 mg/kg body weight); Group $\mathrm{U}(\mathrm{n}=8)$, or untreated group, received the vehicle only. The group of mice $(n=9)$ that were neonatally administrated with the solvent only, were designated as group $\mathrm{C}$, received no treatment at all and served as a blank control. The doses of these drugs were determined based on our previous studies and other studies (10,14-18).

After the 3 weeks of drug treatment, the final hotplate test was performed for all mice with induced adenomyosis and the body weight was also recorded. All mice were anaesthetized and perfused via the ascending aorta with warm $\left(37^{\circ} \mathrm{C}\right)$ saline followed by warm solution composed of $4 \%$ paraformaldehyde and $0.2 \%$ picric acid in $0.1 \mathrm{M}$ phosphate buffer. The perfusion was then immediately followed by the same fixative $\left(4^{\circ} \mathrm{C}\right)$. The uteruses were then excised and were post-fixed overnight at room temperature in the same fixation solution, then embedded in paraffin for histopathological examination and immunohistochemical analysis. L6 and S1 dorsal root ganglions (DRGs) were dissected out and the tissues were post-fixed in the same fixative for $90 \mathrm{~min}$ at $4^{\circ} \mathrm{C}$, and were then immersed in $10 \%$ sucrose in $0.1 \mathrm{M}$ phosphate buffer overnight.

The depth of myometrial infiltration of ectopic endometrium was evaluated following the criteria described by Bird et al (19). Briefly, Grade 1 was defined to be the case when penetration of ectopic endometrium to superficial myometrial, Grade 2 as penetration to mid-myometrium and Grade 3 as penetration beyond mid-myometrium. For ease of statistical analysis, Grade 0 of depth was recorded in the complete absence of any ectopic endometrium in the myometrium.

For histological examination, serial 4-mm sections were obtained from each paraffin-embedded tissue block, and then three randomly selected sections were chosen for hematoxylin and eosin (H\&E) staining to confirm pathological diagnosis. Sections were stained with $0.6 \%$ hematoxylin and $0.5 \%$ eosin for $5 \mathrm{~min}$ at room temperature. If endometrial glands and stroma were observed to be displaced in myometrium, a diagnosis of adenomyosis was made.

Hotplate test procedure. The hotplate test is a commonly used method for measuring nociception in rodents (20), and a test of the response threshold to thermal stimuli, representing a form of acute thermal pain (21). In the current study, the hotplate test was performed using a Hot Plate Analgesia Meter (BME-480; Institute of Biomedical Engineering, Chinese Academy of Medical Sciences, Tianjin, China) consisting of a metal plate of $25 \times 25 \mathrm{~cm}$ in size. The surface of the plate can be heated to a constant temperature of $55.0 \pm 0.1^{\circ} \mathrm{C}$ as measured by a built-in digital thermometer, and a plastic cylinder $(20 \mathrm{~cm}$ in diameter, $18 \mathrm{~cm}$ in height) was placed on the hotplate. Mice were brought to the testing room and allowed to acclimatize for $10 \mathrm{~min}$ prior to the test. The latency to respond to thermal stimuli, defined to be the time (in sec) elapsed from the moment when the mouse was inserted inside the cylinder to the point when it licked its hind paws. Each mouse was tested only once in one session. The latency was calculated as the mean of two readings recorded at intervals of $24 \mathrm{~h}$.

Immunohistochemistry. For all experimental groups, $10 \mu$ m-thick sections of the fixed L6 and S1 DRGs were cut in series in a cryostat and mounted on gelatin-coated slides. Slides were stored at $-20^{\circ} \mathrm{C}$ prior to immunostaining. Sections were quenched with $0.3 \% \mathrm{H}_{2} \mathrm{O}_{2}$ for $10 \mathrm{~min}$ and then blocked in $0.3 \%$ Triton $\mathrm{X}-100$ in $0.05 \mathrm{M}$ Tris- $\mathrm{NaCl}$ with $10 \%$ normal goat serum (NGS) for $1 \mathrm{~h}$ at room temperature. Subsequently, sections were incubated with rabbit polyclonal anti-TRPV-1 (1:5,000; cat. no. acc-030; Alomone Labs, Ltd., Jerusalem, Israel), rabbit monoclonal anti-p-p38 MAPK (1:50; cat. no. 4631; Cell Signaling Technology, Inc., Danvers, MA, USA), or monoclonal anti-ERK1/2 (1:100; cat. no. 4376; Cell Signaling Technology, Inc.) in $0.3 \%$ Triton X-100 in $0.05 \mathrm{M}$ Tris- $\mathrm{NaCl}$, including $1 \% \mathrm{NGS}$ at $4^{\circ} \mathrm{C}$ overnight. Subsequently, biotinylated secondary antibody and Supervision Universal (anti-rabbit) Detection Reagent (horseradish peroxidase) 
A

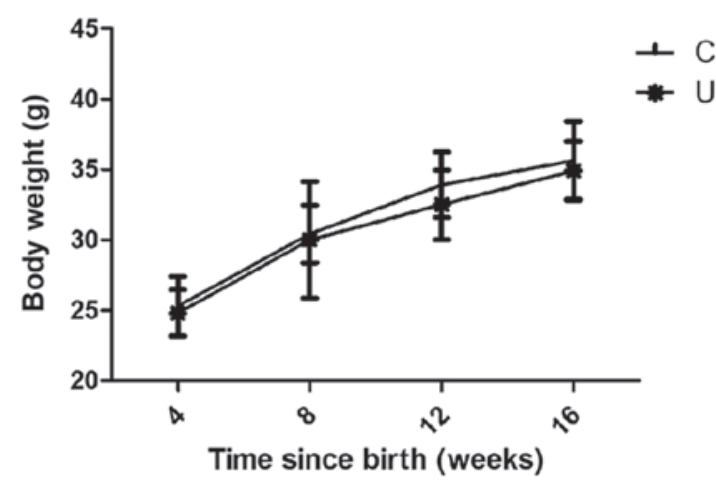

B

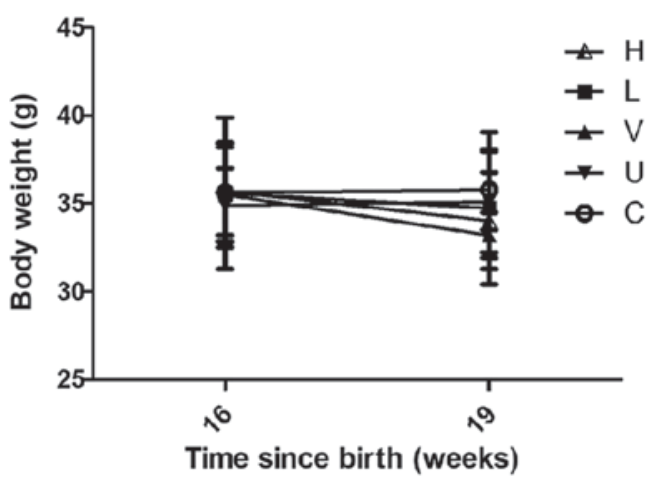

Figure 1. Average body weight in different groups of mice. (A) Prior to quercetin or valproic acid treatment at 16 weeks. (B) Following 3 weeks of quercetin ( $n=9$ mice) or valproic acid ( $n=8$ mice) treatment. H, high-dose quercetin ( $n=9$ mice); L, low-dose quercetin ( $n=9$ mice); V, valproic acid ( $=8$ mice); $\mathrm{U}$, untreated; C, control.

(1:100; cat. no. GK500705; Shanghai Gene Tech Company, Shanghai, China), was incubated at room temperature for $30 \mathrm{~min}$. The bound antibody complexes were stained for 3-5 min or until appropriate for microscopic examination with 3,3'-diaminobenzidineand then counterstained with $0.6 \%$ hematoxylin for $50 \mathrm{sec}$ at room temperature and mounted.

Quantitative analysis was performed according to previous studies (22). Briefly, at least 5 sections from each DRG at each time point were used for quantitative analysis. All mice were sacrificed at the end of the experimental period. The fields were randomly selected in every 3 sections under an Olympus BX51 light microscope (Olympus Corporation, Tokyo, Japan). The number of immunostained neuron profiles was divided by the total number of neuron profiles, and the percentage of labeled neuron profiles was determined. To determine the percentage of labeled neuron within a subset of DRG neurons, the neuron profiles with a clear nucleus were counted.

Statistical analysis. The computer package SPSS18.0 for Windows (SPSS, Inc., Chicago, IL, USA) was used for all the analyses. Means \pm standard deviations of experiments are used to describe statistics. Differences between paired groups were tested using Student's t-test and differences between varied treated groups was analyzed using one-way analysis of variance followed by a post hoc least significant difference test for multiple comparisons. $\mathrm{P}<0.05$ was considered to indicate a statistically significant difference.

\section{Results}

Effect of quercetin treatment on body weight. The body weight of all the mice at 4, 8, 12 and 16 weeks after the induction of adenomyosis, and 3 weeks after quercetin and VPA treatment were recorded (Fig. 1). The results showed that the body weight of the mice dosed with tamoxifen appeared to be lower than that of the control mice at 4,8,12 and 16 weeks; however, no statistically significant difference was detected (Fig. 1A). Following treatment with either the high-and low-dose of quercetin or VPA for 3 weeks, the mice had lower body weight compared to the untreated group, but the difference was not significant (Fig. 1B). Additionally, the high-and low-dose of quercetin and VPA treatment caused a body weight loss

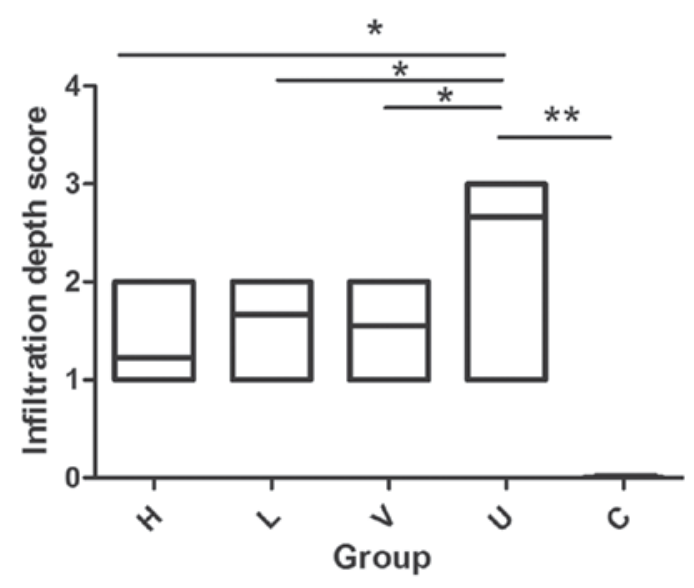

Figure 2. The depth of myometrial infiltration in different groups. Data are expressed as the mean \pm standard deviation. ${ }^{*} \mathrm{P}<0.05,{ }^{* * *} \mathrm{P}<0.01$. H, high-dose quercetin; L low-dose quercetin; V, valproic acid; U, untreated; C, control.

compared to the control group, with no significant difference (Fig. 1B).

Effect of quercetin treatment on the infiltration of myometrium. The effect of quercetin treatment on the myometrium infiltration in mice induced with adenomyosis was evaluated. As the box plots demonstrated in Fig. 2, compared with the control mice, mice dosed with tamoxifen had significantly more infiltration $(\mathrm{P}<0.01)$. Mice treated with either low- or high-dose quercetin or VPA had reduced infiltration compared with the untreated mice dosed with tamoxifen. There was no correlation between dosing with quercetin and the depth of myometrial infiltration $(\mathrm{P}=0.059)$.

Effect of quercetin treatment on the hot plate response latency. In order to determine the response to of mice to noxious thermal stimuli at 4, 8,12, 16 and 19 weeks after birth, a hotplate test was used to investigate changes in response latencies. As demonstrated in Fig. 3A-E, the mice dosed with tamoxifen had significantly decreased response latency compared with control mice $(\mathrm{P}<0.05)$. Before treatment, there was no significant difference in response latency among groups. Compared to group C, a significant difference at 16 weeks and after 
A

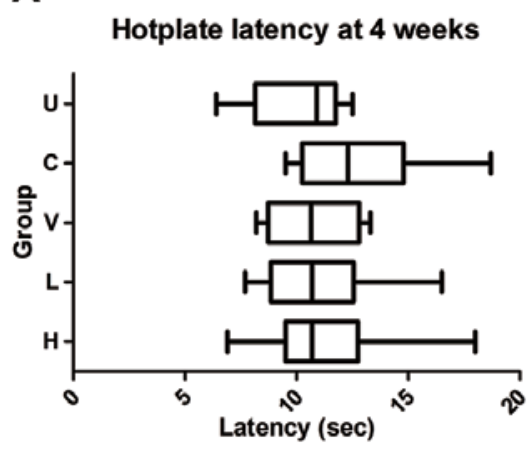

D Hotplate latency at 16 weeks (before treatment)

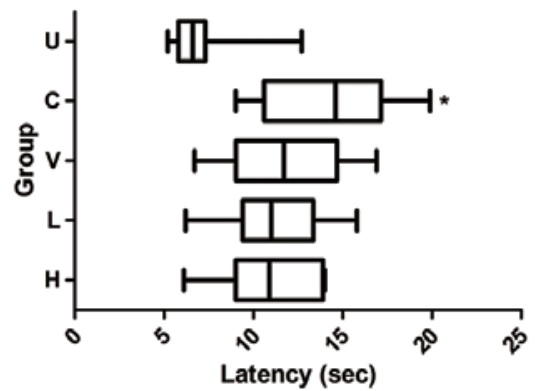

B

Hotplate latency at 8 weeks

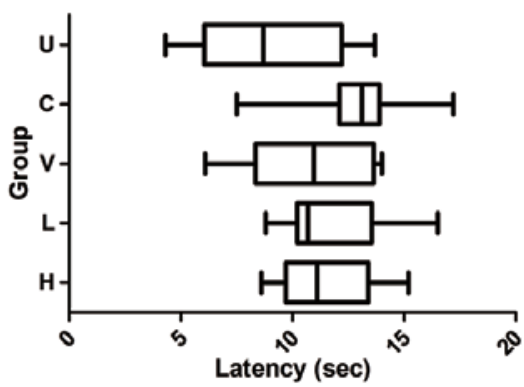

E

\section{Hotplate latency after treatment}

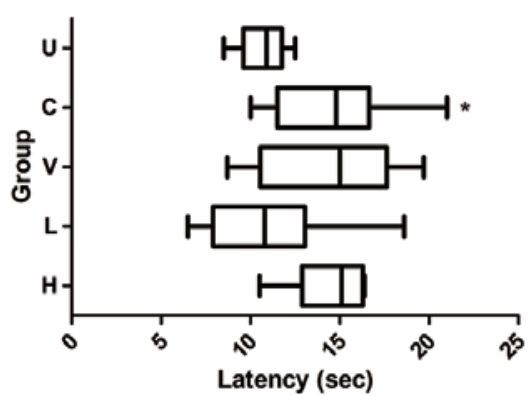

C

Hotplate latency at 12 weeks

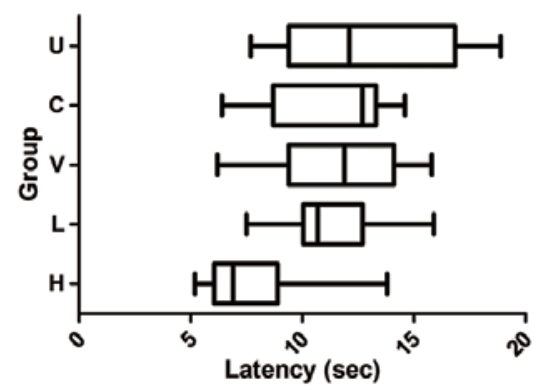

$\mathbf{F}$

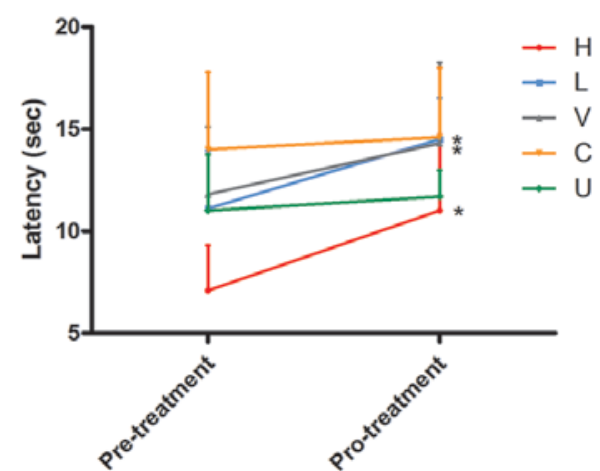

Figure 3. Hotplate response latency in different groups. Hotplate response latency at (A) 4 weeks (B) 8 weeks, (C) 12 weeks, (D) 16 weeks and (E) at the end of treatment. (F) Change of hotplate response latency before (week 16)/after treatment in different groups. Data are expressed as the mean \pm standard deviation. ${ }^{*} \mathrm{P}<0.05$. U, untreated; $\mathrm{C}$, control; $\mathrm{V}$, valproic acid; L low-dose quercetin; H high-dose quercetin.
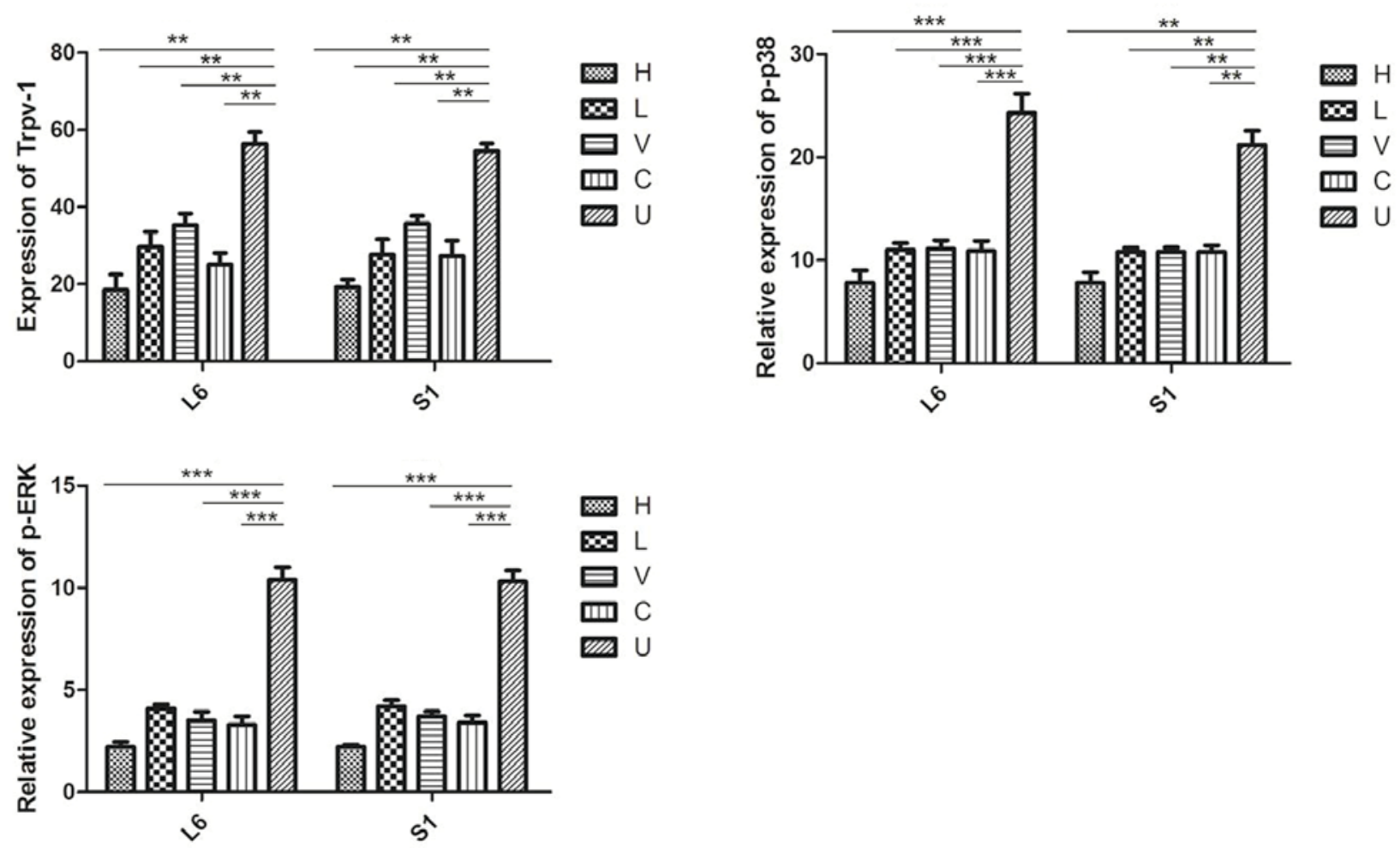

Figure 4. Immunohistochemistry quantification of Trpv-1, p-p38 and p-ERK expression in dorsal root ganglion neurons. ${ }^{* *} \mathrm{P}<0.01$, ${ }^{* * *} \mathrm{P}<0.001$. Trpv-1, transient receptor potential cation channel subfamily V member 1; p-, phospho-; ERK, extracellular signal-regulated kinase. H, high-dose quercetin; L, low-dose quercetin; V, valproic acid; C, control; U, untreated. 
A

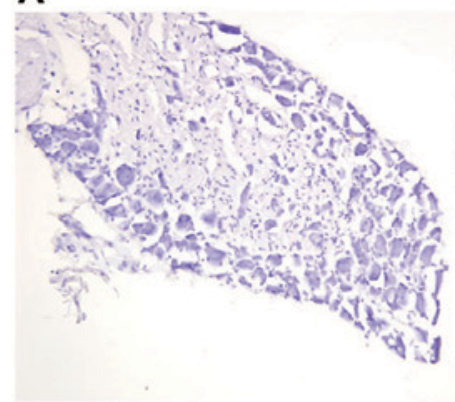

B

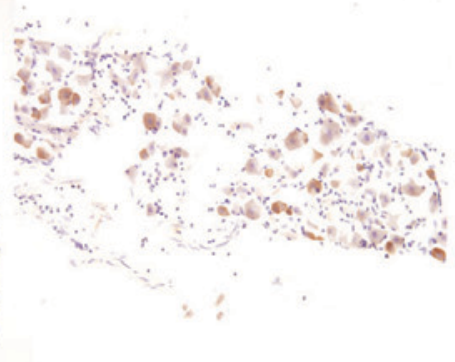

C

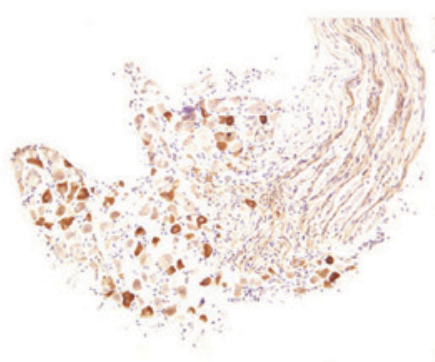

D

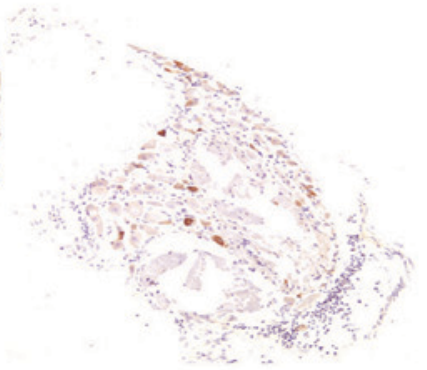

Figure 5. Immunohistochemical staining of L6 dorsal root ganglion for Trpv-1. (A) Negative control using Tris-buffered saline instead of the primary antibody in the from control group. Staining of sections from (B) low-dose quercetin group, (C) untreated group and (D) high-dose quercetin group. Magnification, $\mathrm{x} 400$. Trpv-1, transient receptor potential cation channel subfamily V member 1.

A

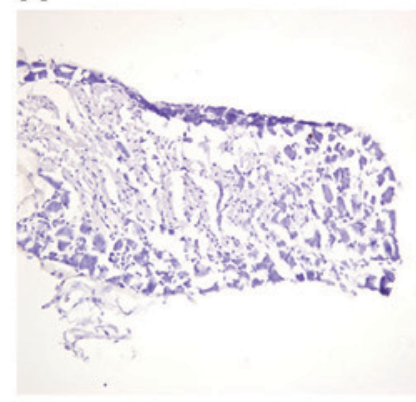

B

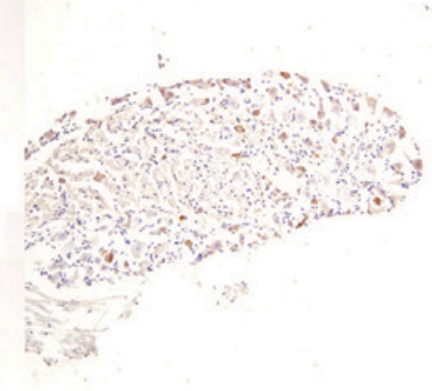

C

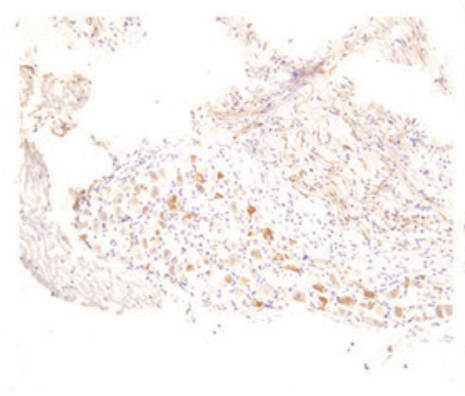

D

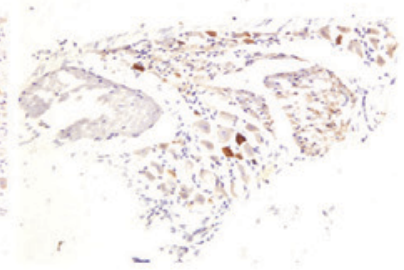

Figure 6. Immunohistochemical staining of L6 dorsal root ganglion for phospho-p38. (A) Negative control using Tris-buffered saline instead of the primary antibody in the L6 DRG from control group. Staining of sections from (B) low-dose quercetin group, (C) untreated group and (D) high-dose quercetin group. Magnification, $\mathrm{x} 400$.

\section{A}

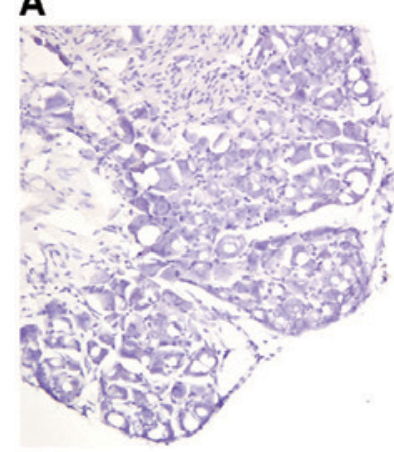

B

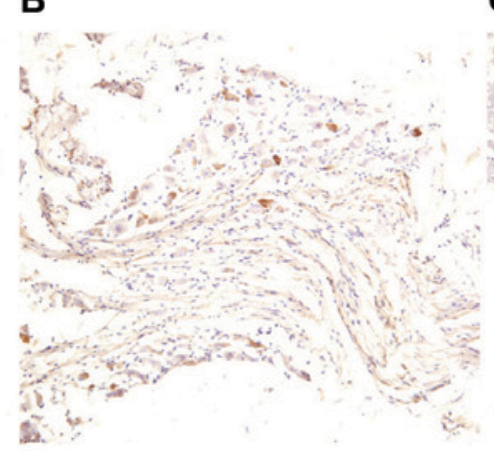

C

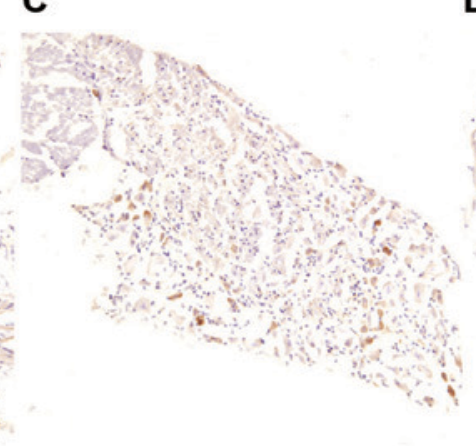

D

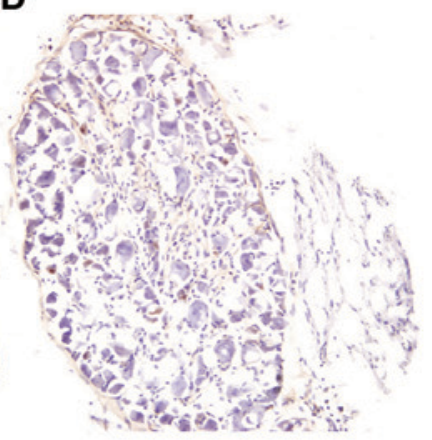

Figure 7. Immunohistochemical staining L6 dorsal root ganglion for p-ERK. (A) Negative control using TBS instead of the primary antibody in the L6 DRG from control group. Staining of sections from (B) low-dose quercetin group, (C) untreated group and (D) high-dose quercetin group. Magnification, $\mathrm{x} 400$. p-ERK, phospho-extracellular signal-regulated kinase.

treatment of groups $\mathrm{U}, \mathrm{V}, \mathrm{L}$ and $\mathrm{H}$ was observed, as presented in Fig. 3D and E. The response latency of the mice of groups $\mathrm{H}$, $\mathrm{L}$ and $\mathrm{V}$, was prolonged following VPA and quercetin treatment at 19 weeks compared to the mice at 16 weeks $(\mathrm{P}<0.05$; Fig. 3F). When compared to the untreated mice, the response latency of the mice either treated with high- or low- quercetin or VPA all exhibited significantly prolonged response latency $(\mathrm{P}<0.05$; Fig. 3F).

Effect of quercetin treatment on the level of Trpv-1,p-p38 and $p$-ERK in DRG neurons. Immunohistochemical method was used to detect the level of Trpv-1, p-p38 and p-ERK in DRG neurons as presented in Figs. 4-7. Compared with the control group, there was an obvious increase in Trpv-1, p-p38 and p-ERK-positive cells in L6 and S1 neurons of the untreated group (P<0.01; Fig. 4). In animals dosed with either high- or low-dose quercetin or VPA the number of Trpv-1, p-p38 and p-ERK-positive cells were all significantly lower than that of the untreated group $(\mathrm{P}<0.01$; Fig. 4). There was no significant difference among the VPA/quercetin-treated groups and control group. Immunohistochemical staining demonstrated that the levels of Trpv-1, p-p38 and p-ERK present in DRG 
neurons were significantly higher in mice dosed with tamoxifen compared with the control group (Figs. 4-7). High and low-dose quercetin significantly reduced the expressions of Trpv1, p-p38 and p-ERK compared to untreated group (; $\mathrm{P}<0.01$; Figs. 4-7). High dose quercetin reduced the levels of Trpv1, p-p38 and p-ERK compared to low dose quercetin, however the change was not statistically significant.

\section{Discussion}

Adenomyosis is a common gynecological disease, which is characterized by the presence of endometrial glands and stroma within the myometrium, frequently affecting women aged $30-50$ years $(1,3,4)$. It is generally estimated to affect $5-70 \%$ of women; however, it is difficult to be precisely determined due to multiple factors $(3,23)$. Hysterectomy remains the 'gold standard' and definitive treatment for adenomyosis. Medical treatments for adenomyosis are of great importance and can include symptom relief and potentially increase fertility. Therapeutic strategies include gonadotropin-releasing hormone agonists, oral contraceptives and progestins, which often cause adverse events, thus impacting the long-term use and adherence to the dosing scheme. However, no medical treatment is universally effective for endometriosis and adenomyosis (24). In our previous study, it was reported that VPA inhibited the proliferation of adenomyosis stromal cells to alleviate the generalized hyperalgesia in ICR mice with induced adenomyosis (10).

The treatments for adenomyosis typically follow the principles of endometriosis treatments by aiming to reduce the production of endogenous estrogen or inducing endometrial differentiation with progestins (24). Increasing evidence indicates that endometriosis has similarities with malignant gynecological tumors, including cell invasion, unrestricted growth, angiogenesis, reduced cell apoptosis (25) and the change in p53 expression (26). Thus, therapeutics used to treat malignant tumors may also me useful for the treatment of endometriosis. Quercetin, as the most frequently investigated flavonoid, was previously reported to be a promising component that can prevent lifestyle-associated diseases through antioxidant, anticarcinogenic, anti-inflammatory, antiviral, immunity adjustment, cardiovascular protection abilities, and effects on hypotension and hypoglycemia $(8,27)$. The current study investigated the effect of quercetin treatment on adenomyosis. The results demonstrated that treatment with quercetin or VPA caused no significant body weight loss in the mice induced with adenomyosis, which may be caused by reduced food intake and a pain-suppressed behavior (28). The histopathology examination suggested that both quercetin and VPA partially inhibited the infiltration of myometrium induced by tamoxifen. The hotplate test results demonstrated that the treatment of quercetin and VPA prolonged the response latency to thermal stimuli, revealing that quercetin reduced hyperalgesia.

Over the last decades, researchers have proposed different pathogenetic hypotheses to explain the initiation and development of adenomyosis (24); however, the exact pathogenesis of adenomyosis remains poorly understood. Emerging evidence demonstrated that adenomyosis is associated with the increased expression of pro-inflammatory cytokines, impaired cell-mediated immunity and neo-angiogenesis (29). To date, various cytokines are thought to be associated with adenomyosis have been analyzed. The present study focused on Trpv-1, p-p38 and p-ERK expressed in DRG neurons, which was hypothesized to have a pivotal role in the central sensitization or analgesia process leading to induction and maintenance of adenomyosis. The current study demonstrated that the levels of Trpv-1, p-p38 and p-ERK expressed in DRG neurons were significantly higher in mice dosed with tamoxifen compared with the control mice, and significantly lower after high- or low-dose quercetin treatment compared with the untreated mice. The results suggested that quercetin treatment alleviates the incidence of hyperalgesia in mice with induced adenomyosis by reducing central sensitization.

Together, the results suggested that quercetin may be a promising agent for alleviating generalized hyperalgesia caused by adenomyosis. To the best of our knowledge, the present study is the first to report the ability of quercetin to alleviate hyperalgesia and inhibit myometrial infiltration in mice with induced adenomyosis.

\section{Acknowledgements}

We sincerely thank Professor Sun-wei Guo for assistance with data analysis and experimental design. The present study was supported by grant no. 81571416 (Jichan Nie) from the National Science Foundation of China.

\section{References}

1. Long L, Chen J, Xiong Y, Zou M, Deng Y, Chen L and Wang Z: Efficacy of high-intensity focused ultrasound ablation for adenomyosis therapy and sexual life quality. Int J Clin Exp Med 8: 11701-11707, 2015.

2. Shi JH, Jin L, Leng JH and Lang JH: Expression of potassium channels in uterine smooth muscle cells from patients with adenomyosis. Chin Med J (Engl) 129: 200-205, 2016.

3. Park H, Kim SH, Cho YM, Ihm HJ, Oh YS, Hong SH, Chae HD, Kim CH and Kang BM: Increased expression of nuclear factor kappa-B p65 subunit in adenomyosis. Obstet Gynecol Sci 59: 123-129, 2016.

4. Bergeron C, Amant F and Ferenczy A: Pathology and physiopathology of adenomyosis. Best Pract Res Clin Obstet Gynaecol 20: 511-521, 2006.

5. Imaoka I, Ascher SM, Sugimura K, Takahashi K, Li H, Cuomo F, Simon $\mathrm{J}$ and Arnold LL: MR imaging of diffuse adenomyosis changes after GnRH analog therapy. J Magn Reson Imaging 15: 285-290, 2002.

6. He Y, He Z, He F and Wan H: Determination of quercetin, plumbagin and total flavonoids in Drosera peltata Smith var. glabrata Y.Z.Ruan. Pharmacogn Mag 8: 263-267, 2012.

7. Chen X: Protective effects of quercetin on liver injury induced by ethanol. Pharmacogn Mag 6: 135-141, 2010.

8. Li Y, Yao J, Han C, Yang J, Chaudhry MT, Wang S, Liu H and Yin Y: Quercetin, Inflammation and Immunity. Nutrients 8: 167, 2016.

9. Han M, Song Y and Zhang X: Quercetin suppresses the migration and invasion in human colon cancer Caco-2 cells through regulating Toll-like receptor 4/nuclear factor-kappa B pathway. Pharmacogn Mag 12 (Suppl 2): S237-S244, 2016.

10. Liu X and Guo S W: Valproic acid alleviates generalized hyperalgesia in mice with induced adenomyosis. J Obstet Gynaecol Res 37: 696-708, 2011.

11. Green AR, Styles JA, Parrott EL, Gray D, Edwards RE, Smith AG, Gant TW, Greaves P, Al-Azzawi F and White IN: Neonatal tamoxifen treatment of mice leads to adenomyosis but not uterine cancer. Exp Toxicol Pathol 56: 255-263, 2005.

12. Parrott E, Butterworth M, Green A, White IN and Greaves P: Adenomyosis-a result of disordered stromal differentiation. Am J Pathol 159: 623-630, 2001. 
13. National Research Council: Guide for the Care and use of Laboratory Animals. National Academies Press, Washington, DC, 1996.

14. Reagan-Shaw S, Nihal M and Ahmad N: Dose translation from animal to human studies revisited. FASEB J 22: 659-661, 2008.

15. Carvalho KM, Morais TC, de Melo TS, de Castro Brito GA de Andrade GM, Rao VS and Santos FA: The natural flavonoid quercetin ameliorates cerulein-induced acute pancreatitis in mice. Biol Pharm Bull 33: 1534-1539, 2010.

16. Mao X, Wang Y, Carter AV, Zhen X and Guo SW: The retardation of myometrial infiltration, reduction of uterine contractility, and alleviation of generalized hyperalgesia in mice with induced adenomyosis by levo-tetrahydropalmatine (1-THP) and andrographolide. Reprod Sci 18: 1025-1037, 2011.

17. Liu X, Pan L, Gong Q and Zhu Y: Leonurine (SCM-198) improves cardiac recovery in rat during chronic infarction. Eur J Pharmacol 649: 236-241, 2010.

18. Yoshino S, Hara A, Sakakibara H, Kawabata K, Tokumura A, Ishisaka A, Kawai Y and Terao J: Effect of quercetin and glucuronide metabolites on the monoamine oxidase-A reaction in mouse brain mitochondria. Nutrition 27: 847-852, 2011.

19. Bird CC, McElin TW and Manalo-Estrella P: The elusive adenomyosis of the uterus-revisited. Am J Obstet Gynecol 112: 583-593, 1972

20. Le Bars D, Gozariu M and Cadden SW: Animal models of nociception. Pharmacol Rev 53: 597-652, 2001.

21. Bannon AW and Malmberg AB: Models of nociception: Hot-plate, tail-flick, and formalin tests in rodents. Curr Protoc Neurosci Chapter 8: Unit 8 9, 2007.
22. Li KC, Wang F, Zhong YQ, Lu YJ, Wang Q, Zhang FX, Xiao HS, Bao L and Zhang X: Reduction of follistatin-like 1 in primary afferent neurons contributes to neuropathic pain hypersensitivity. Cell Res 21: 697-699, 2011

23. Garavaglia E, Audrey S, Annalisa I, Stefano F, Iacopo T, Laura C and Massimo C: Adenomyosis and its impact on women fertility. Iran J Reprod Med 13: 327-336, 2015.

24. Tsui KH, Lee WL, Chen CY, Sheu BC, Yen MS, Chang TC and Wang PH: Medical treatment for adenomyosis and/or adenomyoma. Taiwan J Obstet Gynecol 53: 459-465, 2014.

25. Ueda M, Yamashita Y, Takehara M, Terai Y, Kumagai K, Ueki K, Kanda K, Yamaguchi H, Akise D, Hung YC and Ueki M: Survivin gene expression in endometriosis. J Clin Endocrinol Metab 87: 3452-3459, 2002.

26. Nezhat F, Cohen C, Rahaman J, Gretz H, Cole P and Kalir T: Comparative immunohistochemical studies of bcl-2 and p53 proteins in benign and malignant ovarian endometriotic cysts. Cancer 94: 2935-2940, 2002

27. Nishimuro H, Ohnishi H, Sato M, Ohnishi-Kameyama M, Matsunaga I, Naito S, Ippoushi K, Oike H, Nagata T, Akasaka H, et al: Estimated daily intake and seasonal food sources of quercetin in Japan. Nutrients 7: 2345-2358, 2015.

28. Stevenson GW, Bilsky EJ and Negus SS: Targeting pain-suppressed behaviors in preclinical assays of pain and analgesia: Effects of morphine on acetic acid-suppressed feeding in C57BL/6J mice. J Pain 7: 408-416, 2006.

29. Guo J, Chen L, Luo N, Li C, Chen R, Qu X, Liu M, Kang L and Cheng Z: LPS/TLR4-mediated stromal cells acquire an invasive phenotype and are implicated in the pathogenesis of adenomyosis. Sci Rep 6: 21416, 2016. 